\title{
Under-diagnosis of SARS-CoV-2 infections among children aged 0-15 years, a nationwide seroprevalence study, Israel, January 2020 to March 2021
}

Victoria Indenbaum ${ }^{1, *}$, Yaniv Lustig ${ }^{1,2, *}$, Ella Mendelson ${ }^{1,2}$, Yael Hershkovitz $^{3}$, Aharona Glatman-Freedman ${ }^{2,3}$, Lital KeinanBoker ${ }^{3,4}$, Ravit Bassal2,3

1. Central Virology Laboratory, Ministry of Health, Ramat-Gan, Israel

2. Department of Epidemiology and Preventive Medicine, School of Public Health, Sackler Faculty of Medicine, Tel Aviv University, Tel Aviv, Israel

3. Israel Center for Disease Control, Ministry of Health, Sheba Medical Center, Ramat-Gan, Israel

4. School of Public Health, University of Haifa, Haifa, Israel

* These authors contributed equally to this article and share first authorship

Correspondence: Victoria Indenbaum (Viki.Indenbaum@sheba.health.gov.il)

Until recently, children and adolescents were not eligible for COVID-19 vaccination. They may have been a considerable source of SARS-CoV-2 spread. We evaluated SARS-CoV-2 IgG antibody seroprevalence in Israeli children aged 0-15 years from January 2020 to March 2021. Seropositivity was 1.8-5.5 times higher than COVID-19 incidence rates based on PCR testing. We found that SARS-CoV-2 infection among children is more prevalent than previously thought and emphasise the importance of seroprevalence studies to accurately estimate exposure.

The coronavirus disease (COVID-19) pandemic has spread globally since December 2019. Despite global evidence that children infected with severe acute respiratory syndrome coronavirus 2 (SARS-CoV-2) are more likely to manifest mild symptoms and are at a lower risk of developing severe respiratory disease, there is growing evidence of post COVID-19 condition among children and adolescents [1,2]. Available data on the transmissibility of SARS-CoV-2 among children are contradictory [3]. Moreover, the substantial percentage of asymptomatic infections makes it difficult to accurately estimate the incidence of SARS-CoV-2 infections among children and adolescents [4].

We present results from a nationwide serological study of children aged 0-15 years in Israel. Our aim was to estimate seropositivity during the COVID-19 pandemic, to evaluate the association between the reported confirmed cases and seropositivity rate and to identify the children's odds for SARS-CoV-2 exposure.
COVID-19 vaccination in 12-16-year-olds The rollout of COVID-19 vaccination among adolescents in Israel started in June 2021. By 3 November 2021, $57 \%$ of adolescents (aged 12-16 years) in Israel had received their first vaccine dose and $46 \%$ had received their second vaccine dose [5]. As at 22 November 2021, the COVID-19 vaccine has been approved for 5-11-yearolds in Israel. As a result, a considerable number of children under the age of 16 years in Israel are thought to be susceptible to SARS-CoV-2 infection.

\section{Seroprevalence study}

We studied 2,765 serum samples collected between 1 January 2020 and 31 March 2021 from children aged $0-15$ years, with a median age of 8.9 years (interquartile range: 4.1-13.1). Samples were obtained anonymously from individuals who performed routine or diagnostic blood tests in one of five laboratories located throughout Israel by the Israel National Serum Bank (INSB) [6]. None of the children had received a COVID-19 vaccine. Demographic data of the study cohort are presented in Table 1.

The samples were collected from all seven districts of Israel (Jerusalem, North, Haifa, Judea and Samaria, Central, Tel Aviv and South) and represented children from different regions, socio-economic status and population groups. The data on socio-economic status were allocated to each participant based on their address of residence using the socio-economic residential classification and was divided into low (clusters 1-5) and high (clusters 6-10) [7]. 
SARS-CoV-2 seropositivity and cumulative incidence rate in children aged 0-15 years, Israel, January 2020-March 2021

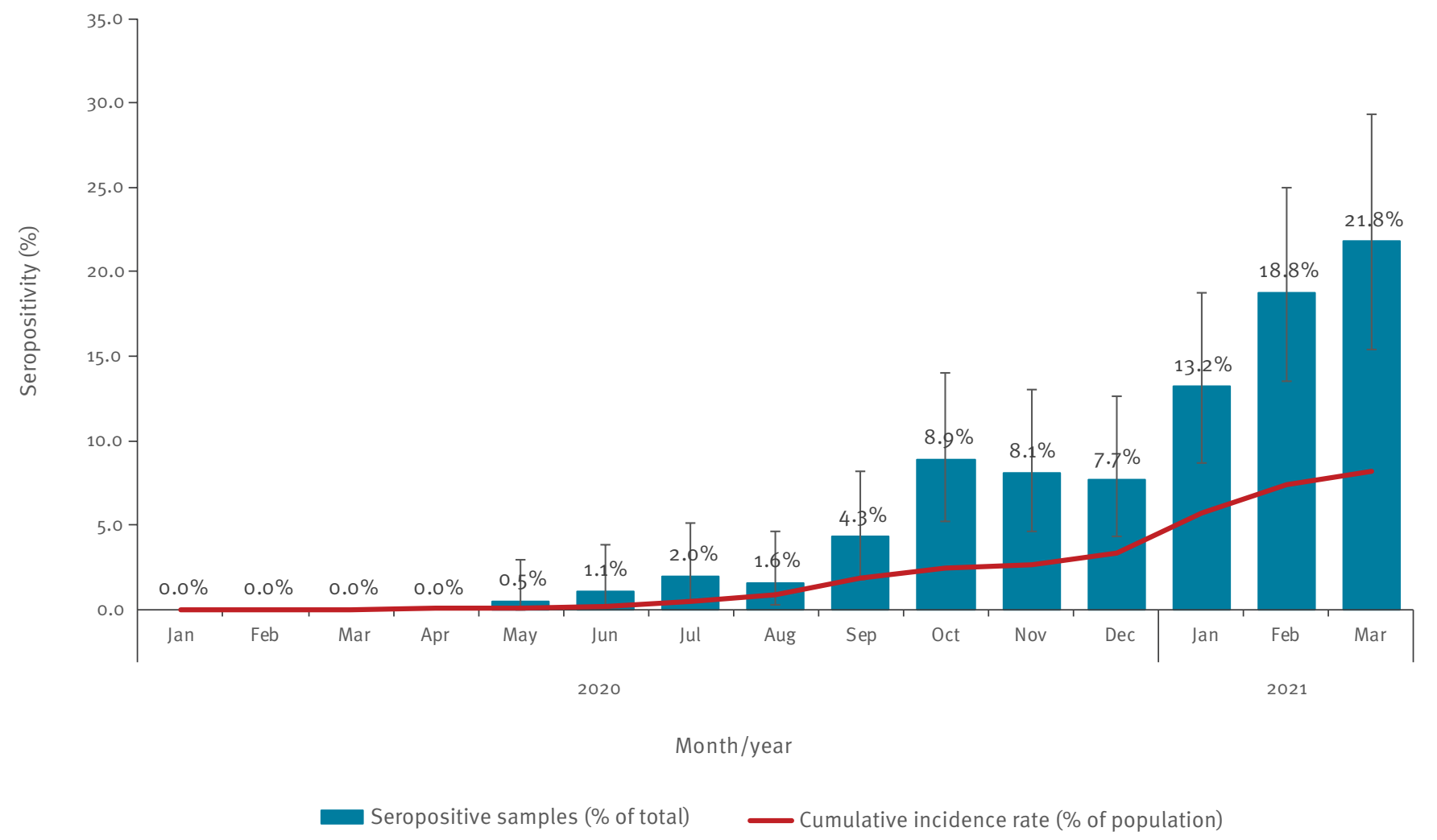

Percentages of SARS-CoV-2 positive serum samples (blue boxes) and cumulative SARS-CoV-2 incidence rate in children (red line) from January 2020 to March 2021. Bars represent $95 \%$ confidence intervals.

All samples were initially screened for SARS-CoV-2 specific IgG antibodies using an in-house ELISA based on the receptor-binding domain assay [8]. In this assay, a sample-to cut-off ratio (S/CO) equal to or above 1.1 is considered to be positive and below, negative, with $88 \%$ sensitivity and $98 \%$ specificity $[8,9]$. To increase sensitivity and specificity, we tested 76 samples with a S/CO below 1.1 using a SARS-CoV-2 pseudo-virus neutralisation assay (NA) [10]. While all 47 samples below 0.8 S/CO were negative, seven samples with a S/CO of $0.8-1.1$ were positive, suggesting that there was no reason to test samples below 0.8 with the confirmatory NA. Therefore, all samples with a S/CO of 0.8 or above were tested using NA. Levels of 16 or above were considered neutralising and thus, seropositive. Levels below 16 were considered not to be neutralising and hence, negative.

\section{Seropositivity rates analysis}

The number, percentage and 95\% confidence interval (Cl) of seropositive individuals in general and by certain characteristics are presented in Table 1 . Overall, $5.6 \%(95 \% \mathrm{Cl}: 4.8-6.6 ; \mathrm{n}=156)$ of samples were seropositive. No statistically significant difference was found among different age and population groups.
Seropositivity rate was $11 \%(95 \% \mathrm{Cl}: 6.9-16.3)$ in the Jerusalem district, significantly higher than in other districts. Lower socio-economic status was associated with significantly higher seropositivity; $7.2 \%$ (95\% Cl: 6.0-8.7) of low socio-economic status children and $3.3 \%$ (95\% Cl: $2.3-4.7$ ) of high socio-economic status children were seropositive.

The Figure demonstrates the percentage of seropositivity over time during the study observation period.

A positive association between seropositivity and time was observed. Specifically, no seropositive samples were observed between January 2020 and March 2020; the percentage of seropositive samples started to rise in May 2020 and reached 21.8\% (95\% Cl: 15.4-29.3) in March 2021, the last month of the study period. We compared the cumulative incidence rate of SARS-CoV-2 positive children confirmed by RT-PCR as documented in the national SARS-CoV-2 repository [5] and the percentage of seropositive samples detected during the study period (Figure). From June 2020 to March 2021, seropositivity was between 1.8 and 5.5 times higher than the cumulative SARS-CoV-2 incidence rate. 
Demographic data and seropositivity of children aged 0-15 years, Israel, January 2020-March 2021 $(\mathrm{n}=2,765)$

\begin{tabular}{|c|c|c|c|c|c|c|}
\hline \multirow{2}{*}{ Characteristics } & \multicolumn{2}{|c|}{ Study participants } & \multicolumn{4}{|c|}{ Seropositivity } \\
\hline & $\mathrm{n}$ & $\%$ & $n$ & $\%$ & $95 \% \mathrm{Cl}$ & $\mathrm{p}$ value \\
\hline Total n & 2,765 & 100 & 156 & 5.6 & $4.8-6.6$ & NA \\
\hline \multicolumn{7}{|l|}{ Age } \\
\hline Median age (IQR) & \multicolumn{2}{|c|}{$8.93(4.1-13.1)$} & & \multicolumn{2}{|c|}{ NA } & \\
\hline \multicolumn{7}{|l|}{ Age group } \\
\hline$<6$ months & 80 & 2.9 & 6 & 7.5 & $2.8-15.6$ & \multirow{6}{*}{0.406} \\
\hline $6-12$ months & 63 & 2.3 & 1 & 1.6 & $0.0-8.5$ & \\
\hline $1-4$ years & 729 & 26.4 & 34 & 4.7 & $3.2-6.5$ & \\
\hline $5-9$ years & 685 & 24.8 & 38 & 5.6 & $4.0-7.5$ & \\
\hline $10-11$ years & 314 & 11.4 & 19 & 6.0 & $3.7-9.3$ & \\
\hline $12-15$ years & 894 & 32.3 & 58 & 6.5 & $5.0-8.3$ & \\
\hline \multicolumn{7}{|l|}{ Sex } \\
\hline Male & 1,436 & 51.9 & 82 & 5.7 & $4.6-7.0$ & \multirow{2}{*}{0.871} \\
\hline Female & 1,329 & 48.1 & 74 & 5.6 & $4.4-6.9$ & \\
\hline \multicolumn{7}{|l|}{ Ethnicity } \\
\hline Jewish and others ${ }^{a}$ & 1,960 & 72.5 & 104 & $5 \cdot 3$ & $4.4-6.4$ & \multirow{2}{*}{0.091} \\
\hline Arab & 742 & 27.5 & 52 & 7.0 & $5.4-9.1$ & \\
\hline \multicolumn{7}{|l|}{ District } \\
\hline Jerusalem & 191 & 6.9 & 21 & 11.0 & $6.9-16.3$ & \multirow{7}{*}{0.001} \\
\hline North & 489 & 17.7 & 26 & 5.3 & $3.5-7.7$ & \\
\hline Haifa & 336 & 12.2 & 16 & 4.8 & $2.8-7.6$ & \\
\hline Judea and Samaria & 170 & 6.2 & 16 & 9.4 & $5.5-14.8$ & \\
\hline Central & 599 & 21.7 & 32 & 5.3 & $3.7-7.5$ & \\
\hline Tel Aviv & 225 & 8.1 & 17 & 7.6 & $4.5-11.8$ & \\
\hline South & 755 & 27.3 & 28 & 3.7 & $2.5-5.3$ & \\
\hline \multicolumn{7}{|l|}{ SES } \\
\hline Median SES (range) & \multicolumn{2}{|c|}{$5(1-10)$} & \multicolumn{4}{|c|}{ NA } \\
\hline High SES $(6-10)$ & 899 & 32.5 & 30 & 3.3 & $2.3-4.7$ & \multirow{3}{*}{$<0.001$} \\
\hline Low SES (1-5) & 1,475 & 53.3 & 107 & 7.2 & $6.0-8.7$ & \\
\hline Unknown SES & 391 & 14.1 & 19 & 4.9 & $3.0-7.5$ & \\
\hline
\end{tabular}

$\mathrm{Cl}$ : confidence interval; IQR: interquartile range; NA: not applicable; SES: socio-economic status.

${ }^{a}$ Brazilian, Belarusian, Chilean, Circassian, Ethiopian, Finnish, Hungarian, Indian, Russian, Ukrainian ( $\mathrm{n}=40$ samples).

\section{Association between population characteris- tics and SARS-CoV-2 seropositivity}

The associations between seropositivity and risk factors as determined by univariate analysis were examined using logistic regression to calculate $p$ values, odds ratios (OR) and $95 \% \mathrm{Cl}$. The results presented in Table 2 show that children living in the Jerusalem district had higher odds (OR: 2.2; 95\% Cl: 1.2-4.0) of being SARSCoV-2-seropositive than children living in the Northern district, and children of low socio-economic status had higher odds (OR: 2.3 ; $95 \% \mathrm{Cl}: 1.5-3.4$ ) of being SARSCoV-2-seropositive than children from high socioeconomic status. There were no significant differences in seropositivity between different age groups, sex and ethnic groups.

\section{Ethical statement}

These data were collected as part of surveillance by the Israel Ministry of Health and thus require no ethical clearance. Additionally, the serosurvey is based on the INSB samples which are anonymous.

\section{Discussion}

In May 2021, the United States (US) Food and Drug Administration (FDA) and the European Medicines Agency (EMA) approved the use of the BNT162b2 mRNA COVID-19 vaccine (Pfizer, New York, US and BioNTech, Mainz, Germany) for children aged $12-15$ years $[11,12]$. On 29 October 2021, the authorisation was expanded by the FDA to include younger children aged 5-11 years with a smaller vaccine dose [13]. The EMA also recommended an extension of indication for the BNT162 b2 mRNA COVID-19 vaccine to include use in children aged 5-11 years on 25 November 2021 [14]. However, to date, vaccine uptake by children and adolescents is progressing slowly worldwide. As a result, people under the age of 16 years remain susceptible to SARSCoV-2 infection and a source for continued circulation. 


\section{TABLE 2}

Association between population characteristics and SARSCoV-2 seropositivity, Israel, January 2020-March 2021 $(\mathrm{n}=156)$

\begin{tabular}{|c|c|c|}
\hline \multirow{2}{*}{ Characteristics } & \multicolumn{2}{|c|}{ Seropositivity } \\
\hline & OR $(95 \% \mathrm{Cl})$ & $p$ value \\
\hline \multicolumn{3}{|l|}{ Age group } \\
\hline <6 months & $5.0(0.6-42.9)$ & 0.140 \\
\hline $6-12$ months & Ref & NA \\
\hline $1-4$ years & $3.0(0.4-22.5)$ & 0.278 \\
\hline $5-9$ years & $3.6(0.5-27.0)$ & 0.206 \\
\hline 10-11 years & $4.0(0.5-30.4)$ & 0.181 \\
\hline $12-15$ years & $4.3(0.6-31.6)$ & 0.152 \\
\hline \multicolumn{3}{|l|}{ Sex } \\
\hline Male & $1.0(0.7-1.4)$ & 0.871 \\
\hline Female & Ref & NA \\
\hline \multicolumn{3}{|l|}{ Ethinicity } \\
\hline Jewish and others ${ }^{a}$ & Ref & NA \\
\hline Arab & $1.3(1.0-1.9)$ & 0.091 \\
\hline \multicolumn{3}{|l|}{ District } \\
\hline Jerusalem & $2.2(1.2-4.0)$ & 0.010 \\
\hline North & Ref & NA \\
\hline Haifa & $0.9(0.5-1.7)$ & 0.722 \\
\hline Judea and Samaria & $1.8(1.0-3.5)$ & 0.063 \\
\hline Central & $1.0(0.6-1.7)$ & 0.985 \\
\hline Tel Aviv & $1.5(0.8-2.7)$ & 0.245 \\
\hline South & $0.7(0.4-1.2)$ & 0.176 \\
\hline \multicolumn{3}{|l|}{ SES } \\
\hline Median SES (range) & $5(1-10)$ & NA \\
\hline High SES $(6-10)$ & Ref & \multirow{2}{*}{0.001} \\
\hline Low SES (1-5) & $2.3(1.5-3.4)$ & \\
\hline
\end{tabular}

$\mathrm{Cl}$ : confidence interval; NA: not applicable; OR: odds ratios; Ref: reference; SARS-CoV-2: severe acute respiratory syndrome coronavirus 2; SES: socio-economic status.

a Brazilian, Belarusian, Chilean, Circassian, Ethiopian, Finnish, Hungarian, Indian, Russian, Ukrainian ( $=40$ samples).

Therefore, it is critical to assess the magnitude of undiagnosed infections in this age group.

Our results suggest that, in the general population, most infections in individuals under 16 years of age are undiagnosed. As at May 2020, we observed an increase in the percentage of seropositive samples, from $0.5 \%$ in May 2020 to $21.8 \%$ in March 2021. However, the percentage of PCR-confirmed infections during the same time period was substantially smaller. At the beginning of the pandemic during the first wave (May-June 2020) the seropositivity percentage was 5-5.5 times higher than the percentage of PCR-confirmed cases. This probably resulted from low availability and limitations imposed on PCR testing and monitoring of suspected cases at the beginning of the pandemic. Later on, following a considerable improvement in the availability of PCR testing and monitoring of suspected cases, the difference between the two measurements decreased. Starting in September 2020, a 2-3-fold difference between COVID-19 incidence and seropositivity was observed and was sustained until March 2021. Our observations are in line with accumulative data demonstrating that most children infected with SARS-CoV-2 experience clinically mild disease or remain asymptomatically infected $[15,16]$.

The finding that children living in the Jerusalem district and those with a low socio-economic status had twofold higher odds of being SARS-CoV-2 seropositive as compared with children living in the Northern district and those with a high socio-economic status, respectively, is consistent with population differences found during the pandemic in Israel. Specifically, more than $30 \%$ of the Jerusalem population belongs to the ultraOrthodox community, which was severely affected by the COVID-19 pandemic [17]. This community consists of many large families living in small apartments, resulting in a crowded environment. Most of them have a low socio-economic status. Structural, religious and social-ideological factors directly or indirectly influenced the high SARS-CoV-2 infection rates among the ultra-Orthodox population in Israel [17].

Although this cohort of the Israeli population was limited in number, through this study we found that more than $20 \%$ of children aged $0-15$ years in Israel had been infected with SARS-CoV-2 as at March 2021. The use of cumulative COVID-19 incidence over time allowed us to determine that overall, at least $50 \%$ of SARS-CoV-2 infections in this age group, were undiagnosed between September 2020 and March 2021 based on the difference observed between cumulative incidence and seropositivity. While our results may suggest that natural infection is more prevalent than anticipated asymptomatic infection, especially in younger ages, might result in low and unsustained antibody levels [18].

\section{Conclusion}

This study emphasises the importance of regular seroprevalence studies to estimate the population's immunity to SARS-CoV-2. Such studies enable characterisation of the fraction of the population that still needs to be protected and can facilitate efforts to reach these individuals. Future studies should investigate the impact of the Delta or other variants of concern on asymptomatic vs symptomatic infections in children and adolescents. Serological studies can also serve as a tool in vaccination programmes planned by countries.

\section{Acknowledgements}

We would like to thank Tal Levin, Yara Knaaneh, Shiri Katz Likvornik, Ravit Koren and Osnat Halpern for performing the technical laboratory work; Rita Lokshin for her assistance in sample collection and separation in the Israel Center for Disease Control. We also would like to thank Kamalia Raguimov from Soroka University Medical Center; Ann But from Haifa and Western Galilee HMO Laboratory; Mohammed Elmraanih from Schneider Children's Medical Center; Rutty 
Hofrichter from Mayanei Hayeshua Medical Center and Mohammed A. Odeh from Jerusalem HMO Laboratory for sample collection. YL is a recipient of the Nehemia Rubin Excellence in Biomedical Research, The TELEM Program of Chaim, Sheba Medical Center.

\section{Conflict of interest}

None declared.

\section{Authors' contributions}

VI, YL and RB designed the study. VI, YL, RB, EM and LKB contributed to data collection and creation of data resources. VI, YL, RB, LKB, EM checked and verified the dataset and prepared it for analysis. $\mathrm{YH}$ and RB performed the statistical analysis. VI, YL and RB wrote the manuscript. VI, YL, RB, EM, $\mathrm{YH}, \mathrm{AGF}$ and $\mathrm{LKB}$ reviewed and edited the manuscript. VI, YL and RB supervised the work. All authors had full access to all data and had final responsibility for the decision to submit for publication.

\section{References}

1. Radtke T, Ulyte A, Puhan MA, Kriemler S. Long-term symptoms after SARS-CoV-2 infection in children and adolescents. JAMA. 2021;326(9):869. https://doi.org/10.1001/jama.2021.11880 PMID: 34264266

2. Sudre CH, Murray B, Varsavsky T, Graham MS, Penfold RS, Bowyer RC, et al. Attributes and predictors of long COVID. Nat Med. 2021;27(4):626-31. https://doi.org/10.1038/s41591-021 01292-y PMID: 33692530

3. Zhu Y, Bloxham CJ, Hulme KD, Sinclair JE, Tong ZWM, Steele LE, et al. A meta-analysis on the role of children in severe acute respiratory syndrome coronavirus 2 in household transmission clusters. Clin Infect Dis. 2021;72(12):e1146-53. https://doi. org/10.1093/cid/ciaa1825 PMID: 33283240

4. Almadhi MA, Abdulrahman A, Sharaf SA, AlSaad D, Stevenson NJ, Atkin SL, et al. The high prevalence of asymptomatic SARS-CoV-2 infection reveals the silent spread of COVID-19. Int J Infect Dis. 2021;105:656-61. https://doi.org/10.1016/j. ijid.2021.02.100 PMID: 33647516

5. Isreal Ministry of Health. COVID-19 daily situation report. Ramat-Gan: Isreal Ministry of Health; 2021. [Accessed: 3 Nov 2021]. Available from: https://datadashboard.health.gov.il/ COVID-19/general

6. Bassal R, Cohen D, Green MS, Keinan-Boker L. The Israel national sera bank: methods, representativeness, and challenges. Int J Environ Res Public Health. 2021;18(5):2280. https://doi.org/10.3390/ijerph18052280 PMID: 33668988

7. Israel Central Bureau of Statistics (CBS). Characterization and classification of local authorities by the socio-economic level of the population in 2015. Jerusalem: CBS; 2021. Available from: https://din-online.info/pdf/lst92e.pdf

8. Indenbaum V, Koren R, Katz-Likvornik S, Yitzchaki M, Halpern 0 , Regev-Yochay G, et al. Testing IgG antibodies against the RBD of SARS-CoV-2 is sufficient and necessary for COVID-19 diagnosis. PLoS One. 2020;15(11):e0241164. https://doi. org/10.1371/journal.pone.0241164 PMID: 33227020

9. Oved K, Olmer L, Shemer-Avni Y, Wolf T, Supino-Rosin L, Prajgrod G, et al. Multi-center nationwide comparison of seven serology assays reveals a SARS-CoV-2 nonresponding seronegative subpopulation. EClinicalMedicine. 2020;29:100651. PMID: 33235985

10. Lustig Y, Sapir E, Regev-Yochay G, Cohen C, Fluss R, Olmer L, et al. BNT162 b2 COVID-19 vaccine and correlates of humoral immune responses and dynamics: a prospective, single-centre, longitudinal cohort study in health-care workers. Lancet Respir Med. 2021;9(9):999-1009. https://doi.org/10.1016/S22132600(21)00220-4 PMID: 34224675

11. United States Food and Drug Administration (FDA). Coronavirus (COVID-19) update: FDA authorizes Pfizer-BioNTech COVID-19 vaccine for emergency use in adolescents in another important action in fight against pandemic. Silver Spring: FDA; 2021 Available from: https://www.fda.gov/news-events/pressannouncements/coronavirus-covid-19-update-fda-authorizespfizer-biontech-covid-19-vaccine-emergency-use

12. European Medicine Agency (EMA). First COVID-19 vaccine approved for children aged 12 to 15 in EU. 2021. Amsterdam:
EMA; 2021. Available from: https://www.ema.europa.eu/en/ news/first-covid-19-vaccine-approved-children-aged-12-15-eu

13. United States Food and Drug Administration (FDA). Vaccines and Related Biological Products Advisory Committee October 26, 2021 Meeting Announcement. Silver Spring: FDA; 2021. Available from: https://www.fda.gov/news-events/pressannouncements/fda-authorizes-pfizer-biontech-covid-19vaccine-emergency-use-children-5-through-11-years-age

14. European Medicine Agency (EMA). Comirnaty COVID-19 vaccine: EMA recommends approval for children aged 5 to 11. Amsterdam: EMA; 2021. Available from: https://www. ema.europa.eu/en/news/comirnaty-covid-19-vaccine-emarecommends-approval-children-aged-5-11.

15. Bobrovitz N, Arora RK, Cao C, Boucher E, Liu M, Donnici $C$, et al. Global seroprevalence of SARS-CoV-2 antibodies: A systematic review and meta-analysis. PLoS One. 2021;16(6):e0252617. https://doi.org/10.1371/journal. pone.0252617 PMID: 34161316

16. Brotons P, Launes C, Buetas E, Fumado V, Henares D, de Sevilla MF, et al. Susceptibility to severe acute respiratory syndrome coronavirus 2 infection among children and adults: a seroprevalence study of family households in the Barcelona Metropolitan Region, Spain. Clin Infect Dis. 2021;72(12):e970-7. https://doi.org/10.1093/cid/ciaa1721 PMID: 33180914

17. Zalcberg S, Block SZ. COVID-19 Amongst the Ultra-Orthodox Population in Israel: An Inside Look into the Causes of the High Morbidity Rates. Contemp Jew. 2021;41(1):1-23. https://doi. org/10.1007/S12397-021-09368-0 PMID: 34305203

18. Yang Y, Wang X, Du RH, Zhang W, Si HR, Zhu Y, et al. Serological investigation of asymptomatic cases of SARSCoV-2 infection reveals weak and declining antibody responses. Emerg Microbes Infect. 2021;10(1):905-12. https:// doi.org/10.1080/22221751.2021.1919032 PMID: 33870851

\section{License, supplementary material and copyright}

This is an open-access article distributed under the terms of the Creative Commons Attribution (CC BY 4.0) Licence. You may share and adapt the material, but must give appropriate credit to the source, provide a link to the licence and indicate if changes were made.

Any supplementary material referenced in the article can be found in the online version.

This article is copyright of the authors or their affiliated institutions, 2021. 